\title{
Simplified development of multiplex real-time PCR through master mix augmented by universal fluorogenic reporters
}

\author{
Simon Wadle',2, Michael Lehnert ${ }^{1}$, Friedrich Schuler ${ }^{1,2}$, René Köppel ${ }^{3}$, Annerose Serr ${ }^{4}$, Roland \\ Zengerle ${ }^{1,2,5}$, and Felix von Stetten ${ }^{1,2}$ \\ ${ }^{1}$ Laboratory for MEMS Applications, IMTEK-Department of Microsystems Engineering, University of \\ Freiburg, Freiburg, Germany, ${ }^{2}$ Hahn-Schickard-Institut für Mikroanalysesysteme, Freiburg, Germany, \\ ${ }^{3}$ Food Control Authority of the Canton of Zürich, Zürich, Switzerland, ${ }^{4}$ Department of Medical \\ Microbiology and Hygiene, University Hospital Freiburg, Freiburg, Germany, and ${ }^{5}$ BIOSS-Centre for \\ Biological Signalling Studies, University of Freiburg, Freiburg, Germany
}

BioTechniques 61:123-128 (September 2016) doi 10.2144/000114443

Keywords: multiplex PCR; mediator probe PCR; universal reporter; MRSA; food panel

Supplementary material for this article is available at www.BioTechniques.com/article/114443.

Mediator probe (MP) PCR is a real-time PCR approach that uses standardized universal fluorogenic reporter oligonucleotides (UR) in conjunction with label-free sequence-specific probes. To enable multiplex real-time MP PCR, we designed a set of five optimized URs with different fluorescent labels. Performance of the optimized URs was verified in multiplex real-time MP PCR for the detection of a pentaplex food panel and a quadruplex methicillin-resistant Staphylococcus aureus (MRSA) panel. Results were comparable to corresponding multiplex hydrolysis probe (HP) PCR, also designated as TaqMan PCR. Analyses of MRSA DNA standards and DNA extracted from patient swab samples showed improved lower limits of detection (LoDs) by a factor of 2-5 when using quadruplex real-time MP PCR instead of HP PCR. The novel set of standardized URs we present here simplifies development of multiplex real-time PCR assays by requiring only the design of label-free probes. In the future, real-time PCR master mixes could be augmented with up to five standardized fluorogenic URs, each emitting light at a different wavelength.

Multiplex real-time PCR has become a valuable tool for the discrimination and quantification of multiple nucleic acid sequences present in a sample, since the method helps save reagents and time $(1,2)$. The most commonly used detection techniques for multiplex real-time PCR employ fluorogenic dual-labeled target-specific probes, such as hydrolysis probes (HPs) or molecular beacons $(3,4)$. To detect different targets, each probe must be labeled with a different fluorophorequencher combination, making the development of these probes tedious and expensive. First, fluorescence modification of probes results in high manufacturing costs (5). Second, the fluorescence properties of each targetspecific probe must be tested and optimized during assay development. A compromise between high signal generation efficiencies and low fluorescence signal cross-talk between detection wavelengths must be achieved $(4,6)$.

The disadvantages of dual-labeled, target-specific probes in real-time PCR have been circumvented by using universal sequence-dependent detection (USD) technologies (7). USD relies on fluorogenic oligonucleotides that can be used independently of the target sequence. Once the universal detection oligonucleotide is optimized with regard to the fluorescence signal generation properties, this optimized version can be used in different assays. Mediator probe (MP) PCR is an example of USD (8). Design rules have been published recently for both single and biplex MP PCR $(9,10)$. These rules comprise the design of the label-free

\section{METHOD SUMMARY}

Here, we describe a novel approach for multiplex real-time PCR using a master mix of five differently labeled universal fluorogenic reporters (URs) that can be used with any combination of label-free mediator probes (MPs), which bind to the specific target sequences. The MPs are cleaved during the primer extension step of PCR, releasing specific mediator sequences that bind to the URs with corresponding hybridization sites to enable the detection of the amplified products. 
target-specific MPs, which are cleaved during primer extension, as well as the design of the universal reporter oligonucleotides (URs). The MP cleavage product is extended after binding to the $3^{\prime}$ end of the UR, which leads to a detectable fluorescence signal due to separation of the fluorophore from the quencher in the UR.

To enable simultaneous detection of more than two targets, the set of URs must be extended. Rules that apply to published UR designs for biplex MP PCR have to be adapted to URs with other fluorescent labels to span the range of available fluorescence detection channels in real-time PCR thermocyclers.

Here, we present a set of five URs with different fluorescent labels. The quality of the UR set was first verified for signal generation upon addition of one or more MPs. The set was then validated using two different multiplex real-time MP PCR assays (Figure 1). The first assay is based on a pentaplex HP PCR food panel that discriminates DNA sequences from different animal species potentially present in pâté, a speciality food product originally made from goose or duck (11).

Table 1: Serial dilution of target DNA for food panel mediator probe (MP) and hydrolysis probe (HP) PCR.

\begin{tabular}{|c|c|c|c|c|c|}
\hline \multirow[t]{2}{*}{ Target mix } & \multicolumn{5}{|c|}{ Amount [ng / reaction] } \\
\hline & Turkey & Pork & Duck & Chicken & Goose \\
\hline 1 & 4 & 0 & 64 & 10 & 0.32 \\
\hline 2 & 2 & 32 & 32 & 3.2 & 0 \\
\hline 3 & 0 & 16 & 10 & 1 & 64 \\
\hline 4 & 58.2 & 7.3 & 2.9 & 0.29 & 29 \\
\hline 5 & 32 & 4 & 1 & 0 & 10 \\
\hline 6 & 16 & 2 & 0.32 & 64 & 3.2 \\
\hline 7 & 8 & 1 & 0 & 32 & 1 \\
\hline
\end{tabular}

The 5 different DNA standards were diluted in dilution buffer consisting of $0.2 \times$ TE buffer (Applichem, St. Louis, MO) containing $10 \mathrm{ng} / \mathrm{mL}$ herring sperm DNA (Invitrogen, Carlsbad, CA).

Instead of the five dual-labeled HPs of the original assay, five label-free MPs together with this UR set were used. The same adaptation was done for a second assay, a quadruplex HP PCR for the simultaneous detection of Staphylococcus aureus together with its potential methicillin-resistance genes (mecA and mecC) and a toxin gene (Panton-Valentine leucocidin, PVL) (12).

\section{Materials and methods}

Universal reporter development A UR for multiplex MP PCR must have a high signal-to-noise ratio (SNR) upon MP addition and a high selectivity for binding to its reverse-complementary MP compared to other MPs.

A
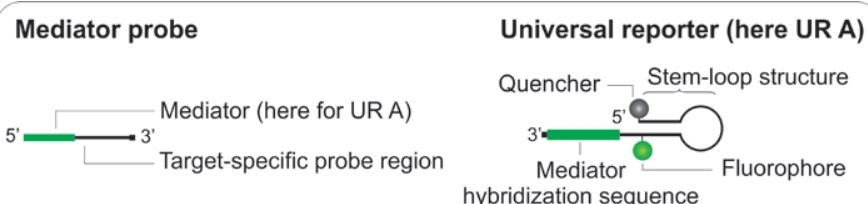

B MP PCR Food panel

\section{Constant set of Universal reporters}

MP PCR MRSA panel
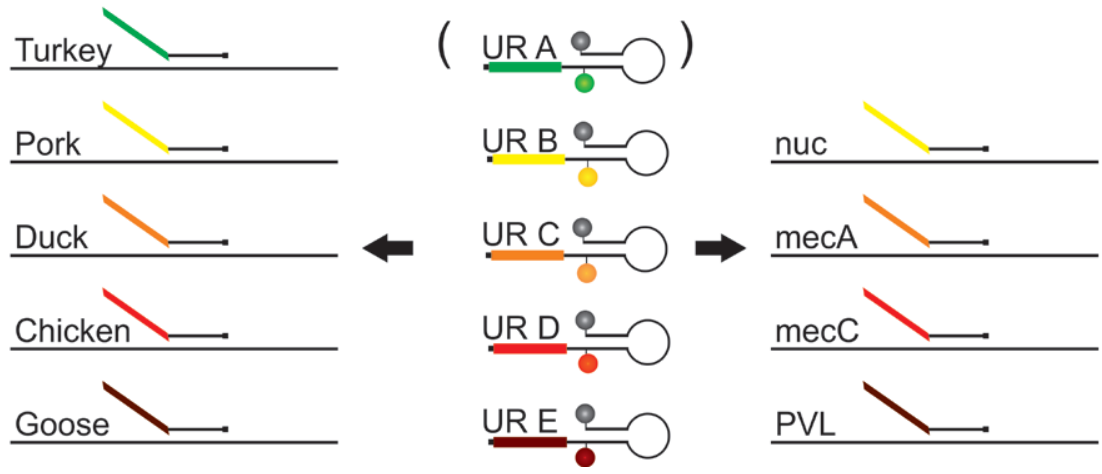

Figure 1. Setup of multiplex real-time mediator probe (MP) PCR. (A) Core components of MP PCR: MP and universal reporter (UR). (B) A single constant set of five URs was used to detect multiple DNA targets (black bars below MP) present in food samples (left) or samples of patients infected with MRSA (right), respectively. A universal set of MP sequences (colored parts) that are attached to the $5^{\prime}$ terminus of the target-specific probe sequences of the MPs are complementary to corresponding binding regions in the URS. The URs are labeled with fluorophores that emit green (FAM), yellow (Rhodamin-6G), orange (ROX), red (Cy5), and deep red (BMN6) light.
To ensure high SNRs, the fluorescence background signal was reduced by placing the fluorophore and quencher moieties in close proximity with the help of a stem-loop structure (Figure 1). Unlike previously published URs, the fluorophores were linked to the nucleotide exactly complementary to the $5^{\prime}$ quencher-modified terminal nucleotide of each of the five URs.

For the FAM-labeled UR A, the effect of guanosine quenching was considered (13-15). Only thymidine and adenosine bases were positioned adjacent and, thus, also opposite the FAM-modified thymidine. Since reports on the quenching of fluorophores other than FAM by nucleotides are contradictory, the effect was not considered in the design of URs B-E.

To ensure binding selectivity toward only one MP, the MP hybridization sequences (MHS) of the URs were randomly generated and showed minimal sequence homology in a BLAST analysis performed with VisualOMP Version 7.8.42.0 (DNA Software, Ann Arbor, MI). A BLAST analysis was also performed for the UR sequences to ensure there was no binding of an extended MP to a UR other than its intended target.

To verify the SNR and selectivity, URs A-E were analyzed in the presence of specific and non-specific MPs according to a previously published procedure (10).

\section{Mediator probe design}

The sequences of primers and probes for food panel and methicillin-resistant Staphylococcus aureus (MRSA) panel detection are based on the literature $(11,12)$. All oligonucleotides were purchased from biomers.net (UIm, Germany) except for primers and probes for the HP PCR-based food detection panel, which were sourced from a commercial kit (Pentaplex Real-Time PCR AllPaté, Microsynth, Switzerland). 
Table 2: Performance of the food panel mediator probe (MP) and hydrolysis probe (HP) PCR.

\begin{tabular}{|c|c|c|c|c|c|c|c|c|c|c|}
\hline \multirow{2}{*}{ Performance parameter } & \multicolumn{2}{|c|}{ Turkey } & \multicolumn{2}{|c|}{ Pork } & \multicolumn{2}{|c|}{ Duck } & \multicolumn{2}{|c|}{ Chicken } & \multicolumn{2}{|c|}{ Goose } \\
\hline & MP & HP & MP & HP & MP & HP & MP & HP & MP & HP \\
\hline PCR efficiency [\%] & $\begin{array}{l}101 \\
\pm 4\end{array}$ & $\begin{array}{r}96 \\
\pm 2\end{array}$ & $\begin{array}{l}80 \\
\pm 2\end{array}$ & $\begin{array}{l}137 \\
\pm 8\end{array}$ & $\begin{array}{r}96 \\
\pm 1\end{array}$ & $\begin{array}{c}100 \\
\pm 4\end{array}$ & $\begin{array}{l}111 \\
\pm 2\end{array}$ & $\begin{array}{l}107 \\
\pm 3\end{array}$ & $\begin{array}{r}98 \\
\pm 1\end{array}$ & $\begin{array}{r}95 \\
\pm 1\end{array}$ \\
\hline$R^{2}$ & 0.98 & 0.99 & 0.99 & 0.97 & 0.99 & 0.99 & 0.99 & 0.99 & 0.99 & 0.99 \\
\hline Max. imprecision $[ \pm \%]$ & 31 & 9 & 14 & 19 & 9 & 13 & 6 & 13 & 14 & 5 \\
\hline Max. inaccuracy $[ \pm \%]$ & 15 & 9 & 10 & 33 & 9 & 8 & 18 & 17 & 5 & 11 \\
\hline
\end{tabular}

Label-free MPs based on the respective HP sequences were easily designed according to recently described guidelines $(11,12)$. Briefly, the 5 'terminal fluorophore of each HP was replaced by a 17-nucleotide generic sequence (18 nucleotides in the case of $\mathrm{MP}_{\mathrm{PVL}}$ ) that is the reversecomplement to the MP hybridization sequence of one of the URs. Instead of the 3'-terminal quencher of the HP, the MP has a C3-spacer to block extension by a polymerase. All oligonucleotide sequences for MP and HP PCR are listed in Supplementary Table S1.

Use of universal reporter oligonucleotides in multiplex PCR

Food panel: Multiplex real-time MP PCR with the novel UR set was compared with reference HP PCR using mixes of DNA (Table 1) extracted from pure samples of turkey, pork, duck, chicken, and goose, or from two different meat samples purchased from a local supermarket (DNA extracted as described in Reference 11).

HP PCR was set up according to the Pentaplex Real-Time PCR AllPaté Kit manual from Microsynth using the QuantiFast Multiplex PCR $+R$ master mix (Qiagen, Hilden, Germany). For MP $\mathrm{PCR}$, primers (200 nM final concentration except for goose primer, which was $100 \mathrm{nM})$, MPs (80 nM), and universal reporter A-E (40 nM) were used with the same master mix. Five microliters of 1 of the 5 DNA target mixes from Table 1 or $5 \mu \mathrm{L}$ of DNA extract from the 2 meat samples (1:10 dilution in DNA dilution buffer) was mixed with $20 \mu \mathrm{L} \mathrm{HP}$ or MP PCR reagents, respectively.
All reactions were run in four technical replicates. PCR thermocycling was performed in a Rotor-Gene $Q$ real-time PCR thermocycler (Qiagen) with the following protocol: $5 \mathrm{~min}$ at $95^{\circ} \mathrm{C}$ for polymerase hot start, then 45 cycles of $5 \mathrm{~s} 95^{\circ} \mathrm{C}, 20 \mathrm{~s} 60^{\circ} \mathrm{C}$. Readout of all five fluorescence channels was performed during the annealing step. The fluorescence readout gain for HP and UR detection was optimized automatically using the Rotor-Gene $Q$ software to reach background fluorescence values of 5-10 RFU (relative fluorescence units) (Supplementary Material). Data analysis was based on $\mathrm{C}_{\mathrm{q}}$ values (Rotor-Gene $\mathrm{Q}$ analysis software $Q$ 2.1.0.9 with slope correction for normalized fluorescence data) determined at a fluorescence threshold that was selected with the second derivation maximum method (16). All $C_{q}$ values $>35$ were excluded from the analysis. $C_{q}$ values were plotted against corresponding input concentrations for all five target DNAs to generate standard curves using Origin 9.0 software (OriginLab Coop., Northampton, MA). For DNA quantification of the two meat samples based on standard curves generated in the reactions with the other target mixes (Table 1), Target Mix 1 was used as the inter-run calibrator.

MRSA panel: As an important performance characteristic of diagnostic assays, the analytical limits of detection (LoDs) of multiplex MP and HP PCR were analyzed using gBlock gene fragments (Integrated DNA Technologies Inc., Leuven, Belgium) (sequences listed in the Supplementary Material) diluted in DNA dilution buffer. QuantiFast Multiplex PCR $+R$ master mix

Table 3: Quantities of meat ${ }^{1}$ in samples analyzed by food panel mediator probe (MP) and hydrolysis probe (HP) PCR.

\begin{tabular}{|c|c|c|c|c|c|c|c|c|c|c|}
\hline \multirow{2}{*}{ Sample } & \multicolumn{2}{|c|}{ Turkey } & \multicolumn{2}{|c|}{ Pork ${ }^{2}$} & \multicolumn{2}{|c|}{ Duck ${ }^{3}$} & \multicolumn{2}{|c|}{ Chicken } & \multicolumn{2}{|c|}{ Goose $^{3}$} \\
\hline & MP & HP & MP & HP & MP & HP & MP & HP & MP & HP \\
\hline Sample 1 & - & - & $>64$ & $>64$ & $<0.32$ & $<0.32$ & - & - & $<0.32$ & $<0.32$ \\
\hline Sample 2 & - & - & $>64$ & $>64$ & 57 & 54 & - & - & 16 & 64 \\
\hline \multicolumn{11}{|c|}{$\begin{array}{l}{ }^{1} \text { Expressed as ng per reaction. } \\
2 \text { The exact amount of pork could not be determined. First, no inter-run calibrator was used. Secondly, the } \\
\text { value is above the quantified range using the serial dilution of DNA shown in Table } 1 . \\
{ }^{3} \text { The exact amount of duck and goose DNA could not be determined in meat sample } 1 \text {. The values are } \\
\text { below the quantified range using the serial dilution of DNA shown in Table } 1 \text {. }\end{array}$} \\
\hline
\end{tabular}

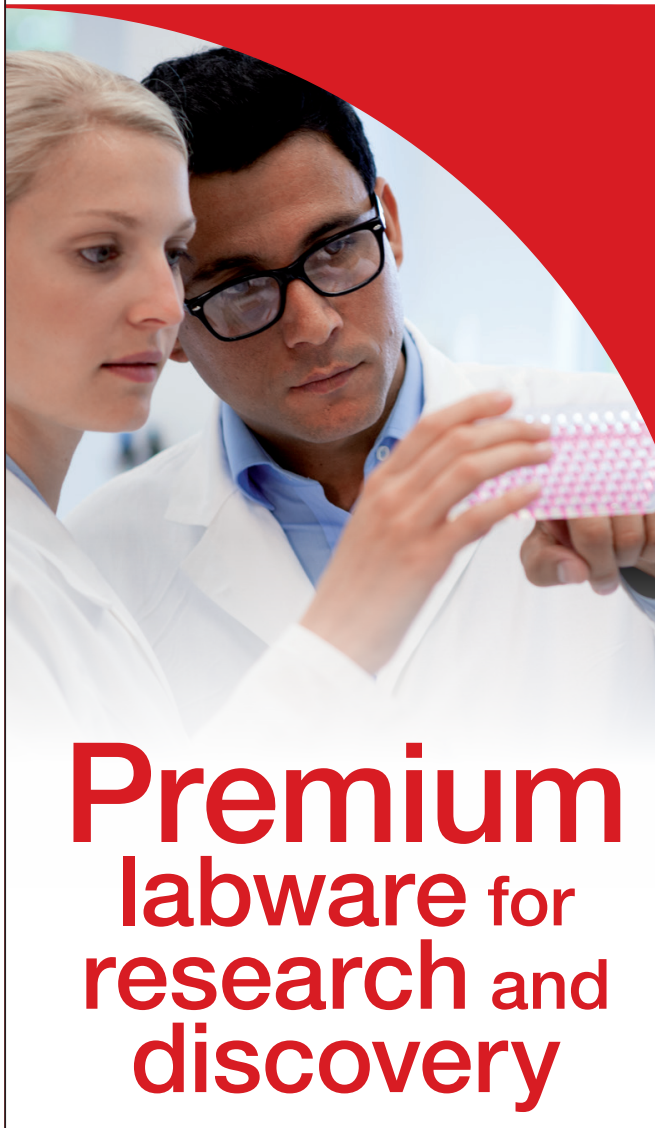

\section{Cell and Tissue Culture}

- Three different color coded growth surfaces

- Optimized, user-friendly geometries

- Labeling of all products with LOT number and expiration date

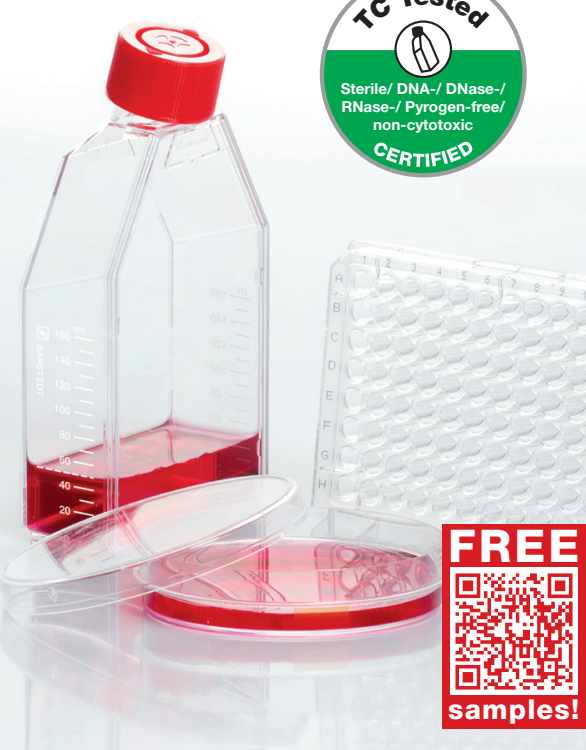


Table 4: Limit of detection (LOD) ${ }^{1}$ of multiplex quadruplex methicillin-resistant Staphylococcus aureus (MRSA) mediator probe (MP) and hydrolysis probe (HP) PCR.

\begin{tabular}{|l|c|c|}
\hline Target & MP PCR & HP PCR \\
\hline nuc (S. aureus marker) & Positive in all cases & Positive in all cases \\
\hline mecA resistance gene & 18 & 99 \\
\hline mecA with $P V L$ present & 33 & 70 \\
\hline mecC resistance gene & 12 & 52 \\
\hline$P V L$ toxin gene & 11 & 51 \\
\hline$P V L$ with $m e c A$ present & 13 & 51 \\
\hline${ }^{1}$ Expressed as copies/reaction with 95\% probability.
\end{tabular}

was supplemented with the nuc gene gBlock gene fragments at 100 copies per reaction, primers (final concentration of $500 \mathrm{nM}$ except for the mecA primer, which was $700 \mathrm{nM})$, and HPs (200 nM) or MPs (200 nM) together with URs B-E (50 nM). One microliter of each single-target DNA or $1 \mu \mathrm{L}$ of a mix of the mecA gene and the $P V L$ gene gBlock gene fragment was used in $15 \mu \mathrm{L}$ reactions at final concentrations of 400, 200, 100, 50, 10, 5, and 1 copies per reaction. All samples were run as six technical replicates. PCR thermocycling was performed in a Rotor-Gene $Q$ with the following protocol: $5 \mathrm{~min} 95^{\circ} \mathrm{C}$ for polymerase hot start, then 45 cycles of 15 s $94^{\circ} \mathrm{C}, 40 \mathrm{~s} 58^{\circ} \mathrm{C}$. Readout was performed as described for the food panel reactions. LoDs were determined using SPSS software version 19 probit analysis (IBM, Armonk, NY) (17) with conditions for positive amplifications as described before (9).

For verification of the assay with clinical samples, MRSA surveillance swabs were collected from anterior nares and processed at the local microbiology department in accordance with the Microbiology Procedures Quality Standards (MiQ) issued by the German Society for Hygiene and Microbiology. Conventional culture was performed on Columbia blood agar and ChromID MRSA (BioMérieux, Marcy-l'Étoile, France) as the commercially available chromogenic MRSA screening agar. For multiplex PCR, DNA was extracted using the QIAamp DNA mini kit (Qiagen) after resuspension of bacteria from the swab samples in $2 \mathrm{~mL}$ PBS. The kit was used according to the manual for isolation of genomic DNA from Grampositive bacteria using lysozyme (SigmaAldrich, Munich, Germany) for lysis. Three microliters of DNA extracts from clinical samples were used in $15 \mu \mathrm{L}$ reactions. The clinical samples were analyzed as four technical replicates. Data were analyzed on the basis of $\mathrm{C}_{\mathrm{q}}$ values as described for the food panel above.

\section{Results and discussion}

Universal reporter development Verification results are presented in the Supplementary Material. SNRs of the 5 URs (A-E) ranged from 21.4-fold to >28-fold signal increase upon MP addition in comparison to no-MP control reactions. A signal increase $>10$ is considered sufficient for signal generation in MP PCR. No nonspecific signal generation and no significant fluorescence cross-talk were detectable. Concerning fluorescence quenching, the UR set showed reduced background fluorescence of 81\%-95\% and 23\%-99\% compared with food panel HPs and MRSA HPs, respectively (Supplementary Material).

Universal reporter oligonucleotide set use in mediator probe PCR for detection of food and MRSA panels For the food panel, most values of multiplex MP PCR compared with multiplex HP PCR from Microsynth are within the acceptable range according to FDA guidelines (www.fda.gov/downloads/ drugs/guidancecomplianceregulatoryinformation/guidances/ucm368107.pdf) as well as within the range of previously published data (11) (Table 2).

Significant differences between the two methods were observed for turkey and pork DNA detection. For the detection of turkey DNA, the MP PCR showed a lower correlation $\left(R^{2}\right)$ between the input DNA concentration and the $\mathrm{C}_{\mathrm{q}}$ values analyzed as well as a higher maximum imprecision and inaccuracy compared with the HP PCR. Considering the standard curves (Supplementary Material), the relatively high imprecision of $31 \%$ and, thus, the low $R^{2}$ were due to a high variance of the $C_{q}$ values between the 4 replicate reactions containing 16 ng turkey DNA. This variance might be primarily due to different levels of nonspecific side reactions in the different replicates.

In the case of pork DNA detection, the HP PCR performed significantly worse than the MP PCR with respect to $R^{2}$, the maximum imprecision, and inaccuracy. Here, the low coefficient of determination, $R^{2}$ of the standard curve, arose from the relatively high inaccuracy in the detection of $1 \mathrm{ng}$ (inaccuracy: 21\%), $4 \mathrm{ng}(22 \%)$, and $7.3 \mathrm{ng}$ (33\%) DNA per reaction (see standard curve in the Supplementary Material). This inaccuracy was also the reason for the implausibly high $\mathrm{PCR}$ efficiency of $137 \%$.

Apart from these differences, the novel MP PCR was found to perform essentially equally to the commercially available HP PCR.

Equal performance of the two detection formats was also observed for the analysis of DNA extracted from meat samples. Both methods were able to detect different DNA sequences present in the different samples (Table 3). The amount of DNA of the different sequences detected is in the same range for both methods. Contradicting the vendors' declarations, significant amounts of pork were detected using both methods. It has been reported previously that the usage

Table 5: $\mathrm{C}_{q}$ values from methicillin-resistant Staphylococcus aureus (MRSA) mediator probe (MP) and hydrolysis probe (HP) PCR for nuc and mecA gene detection of DNA extracted from MRSA patient samples.

\begin{tabular}{|c|c|c|c|c|c|}
\hline \multirow{2}{*}{ Sample } & \multirow{2}{*}{ CFU range on agar plate } & \multicolumn{2}{|c|}{ MP PCR $C_{q}$} & \multicolumn{2}{|c|}{ HP PCR $\mathrm{C}_{q}$} \\
\hline & & nuc & mecA & nuc & mecA \\
\hline 1 & 1 & - & - & - & - \\
\hline 2 & $1-50$ & $34-35$ & 26 & 37-39 (3) & 27 \\
\hline 3 & $1-50$ & $39 \& 42(2)$ & 30 & - & 31 \\
\hline 4 & $1-50$ & $34-37$ & 22 & $39 \& 45(2)$ & 23 \\
\hline 5 & $50-500$ & 29 & 29 & 32 & 30 \\
\hline 6 & $50-500$ & 22 & 22 & 24 & 23 \\
\hline 7 & $50-500$ & 28 & 28 & 30 & 29 \\
\hline 8 & $>500$ & 26 & 27 & 29 & 28 \\
\hline
\end{tabular}

The $C_{q}$ values represent the mean of 4 replicates $\left(C_{q} S D<0.5\right.$ cycles) if not otherwise indicated [e.g., $C_{\text {q }}$ value range of "37-39 (3)"]. The number in brackets indicates the number of positive samples out of the four replicates. CFU: colony forming units. 
of relatively cheap meat such as pork is common in the manufacture of pâté (11); however, with the samples tested here, the relative amount of pork is even higher than the amount of meat the samples are declared to be manufactured from: duck, chicken, and pheasant (Sample 1) and goose (Sample 2), respectively.

For the detection of MRSA, the analytical LoDs of multiplex MP and HP PCR according to a probit analysis are shown in Table 4. MP PCR showed significantly lower LoDs than HP PCR (e.g., for the mecA resistance gene, the MP was 18 copies per reaction compared to 99 copies per reaction for HP). This is primarily due to a higher SNR when using the URs for signal generation compared with the dual-labeled HPs, as shown previously (9). The higher SNR is in turn a function of the $23 \%-99 \%$ reduced fluorescence background of URs compared with HPs (Supplementary Material).

In analyses of DNA extracted from patient swab samples, the HP PCR failed to detect the mecA gene in six samples, whereas MP PCR could detect the mecA gene in all samples, with one exception (Sample 1, Table 5). Reproducible nuc gene detection failed in two out of eight samples (Samples 1 and 3) using MP PCR. In comparison, HP PCR could not reproducibly detect the nuc gene in four out of eight samples. This result reflects the better LoD of multiplex MP PCR discussed above. The mecC gene and the $P V L$ gene were not detected in any of the samples.

Here, we present a novel set of URs for simplified set-up of multiplex real-time PCR. This set was validated for detection using a food panel and a MRSA panel, and compared with the respective HP PCR, which uses five (food panel) or four (MRSA) different dual-labeled HPs. The MP PCR performed equally well in the case of the food panel detection (except for turkey detection) and even better than the reference HP PCR in the case of the MRSA panel.

Multiplex real-time MP PCR is superior to HP PCR in terms of optimization of the fluorogenic molecules for signal generation since the URs do not need to be optimized for the detection of each target. This set of URs can now be used for several multiplex nucleic acid target MP PCRs. For those assays, label-free MPs can be designed either based on already established HP assays or according to the MP design guidelines published previously (10). The UR set could also be pre-stored as lyophilized PCR mixes or by drying the URs into microtiter plates or PCR tubes, similar to the method described in Reference 18. Such universal consumables could be manufactured large-scale, allowing for cost-efficient multiplex real-time PCR.

\section{Author contributions}

S.W. designed and performed experiments, analyzed data, and wrote the manuscript. M.L. performed food panel PCR experiments. F.S. performed MRSA DNA extraction from swabs. R.K. provided DNA extracted from pure meat samples and the extraction protocol for mixed meat samples. A.S. provided pre-characterized patient swab samples. R. Z. and F.v.S. provided Input on experimental design and proofread the manuscript.

\section{Acknowledgments}

This study was funded by the German federal ministry of economic affairs and energy in the SIGNO program as part of the project MultiDart (FKZ 03SHWB047) and in the ZIM program as part of the project MP PCR Desire (FKZ KF2162035CR4) and by the EU framework 7 project AngeLab (FKZ 317635). We thank Franziska Schlenker for support in the laboratory.

\section{Competing interests}

The authors declare no competing interests.

\section{References}

1. Wittwer, C.T., M.G. Herrmann, C.N. Gundry, and K.S. Elenitoba-Johnson. 2001. Real-time multiplex PCR assays. Methods 25:430-442.

2. Mackay, I.M. 2004. Real-time PCR in the microbiology laboratory. Clin. Microbiol. Infect. 10:190212.

3. Juskowiak, B. 2011. Nucleic acid-based fluorescent probes and their analytical potential. Anal. Bioanal. Chem. 399:3157-3176.

4. Kubista, M., J.M. Andrade, M. Bengtsson, A. Forootan, J. Jonak, K. Lind, R. Sindelka, R. Sjoback, et al. 2006. The real-time polymerase chain reaction. Mol. Aspects Med. 27:95-125.

5. Gardner, S.N., T.A. Kuczmarski, E.A. Vitalis, and T.R. Slezak. 2003. Limitations of TaqMan PCR for detecting divergent viral pathogens illustrated by hepatitis $A, B, C$, and E viruses and human immunodeficiency virus. J. Clin. Microbiol. 41:2417-2427.

6. Gaudron, T., Peters, C., Boland, E., Steinmetz, A., Moris, G., 2009. Development of a
quadruplex-real-time-PCR for screening food for genetically modified organisms. Eur Food Res Technol. 229:295-305.

7. Faltin, B., R. Zengerle, and F. von Stetten. 2013. Current methods for fluorescence-based universal sequence-dependent detection of nucleic acids in homogenous assays and clinical applications. Clin. Chem. 59:1567-1582.

8. Faltin, B., S. Wadle, G. Roth, R. Zengerle, and F. von Stetten. 2012. Mediator probe PCR: a novel approach for detection of real-time PCR based on label-free primary probes and standardized secondary universal fluorogenic reporters. Clin. Chem. 58:1546-1556.

9. Wadle, S., M. Lehnert, S. Rubenwolf, R. Zengerle, and F. von Stetten. 2016. Real-time PCR probe optimization using design of experiments approach. Biomolecular Biomol Detect Quantif. 7:1-8.

10. Wadle, S., S. Rubenwolf, M. Lehnert, B. Faltin, M. Weidmann, F. Hufert, R. Zengerle, and F. von Stetten. 2014. Mediator probe PCR: detection of real-time PCR by label-free probes and a universal fluorogenic reporter. Methods Mol. Biol. 1160:55-73.

11. Köppel, R., Daniels, M., Felderer, N., BrünenNieweler, C., 2013. Multiplex real-time PCR for the detection and quantification of DNA from duck, goose, chicken, turkey and pork. Eur Food Res Technol. 236:1093-1098.

12. Pichon, B., R. Hill, F. Laurent, A.R. Larsen, R.L. Skov, M. Holmes, G.F. Edwards, C. Teale, and A.M. Kearns. 2012. Development of a real-time quadruplex PCR assay for simultaneous detection of nuc, Panton-Valentine leucocidin (PVL), mecA and homologue mecALGA251. J. Antimicrob. Chemother. 67:2338-2341.

13. Seidel,C.A., A. Schulz, and M.H. Sauer. 1996. Nucleobase-specific quenching of fluorescent dyes. 1. Nucleobase one-electron redox potentials and their correlation with static and dynamic quenching efficiencies. J Phys Chem. 100:55415553.

14. Marras, S.A, F.R. Kramer, and S. Tyagi. 2002. Efficiencies of fluorescence resonance energy transfer and contact-mediated quenching in oligonucleotide probes. Nucleic Acids Res. 30:e122.

15. Crockett, A.O. and C.T. Wittwer. 2001 Fluorescein-labeled oligonucleotides for real-time pcr: using the inherent quenching of deoxyguanosine nucleotides. Anal. Biochem. 290:89-97.

16. Tichopad, A., M. Dilger, G. Schwarz, and M.W. Pfaffl. 2003. Standardized determination of real-time PCR efficiency from a single reaction set-up. Nucleic Acids Res. 31:e122.

17. Finney, D.J. 1971. Probit Analysis. Cambridge University Press. New York, NY.

18. Rombach, M., D. Kosse, B. Faltin, S. Wadle, G. Roth, R. Zengerle, and F. von Stetten. 2014 Real-time stability testing of air-dried primers and fluorogenic hydrolysis probes stabilized by trehalose and xanthan. Biotechniques 57:151-155.

Received 01 April 2016; accepted 03 June 2016.

Address correspondence to Simon Wadle, HahnSchickard-Institut für Mikroanalysesysteme, Georges-Koehler-Allee 103, 79110 Freiburg, Germany. Email: simon.wadle@hahn-schickard.de

To purchase reprints of this article, contact: biotechniques@fosterprinting.com 